\title{
ON UNIFICATION AND NUCLEON DECAY IN SUPERSYMMETRIC GRAND UNIFIED THEORIES BASED ON $S U(5)$
}

\author{
Ilja Doršner $^{1}: *$ Pavel Fileviez Pérez $^{2}+\dagger$ and Germán Rodrigo 34 \\ ${ }^{1}$ The Pennsylvania State University \\ 104 Davey Lab, PMB 025, \\ University Park, PA 16802 \\ ${ }^{2}$ Centro de Física Teórica de Partículas \\ Departamento de Física. Instituto Superior Técnico \\ Avenida Rovisco Pais 1, 1049-001 Lisboa, Portugal \\ ${ }^{3}$ Instituto de Física Corpuscular, \\ CSIC-Universitat de València \\ Apartado de Correos 22085, \\ E-46071 Valencia, Spain
}

(Dated: March 22, 2021)

\begin{abstract}
We investigate the unification constraints in the minimal sypersymmetric grand unified theories based on $S U(5)$ gauge symmetry. The most general constraints on the spectrum of minimal supersymmetric $S U(5)$ and flipped $S U(5)$ are shown. The upper bound on the mass of the colored Higgs mediating proton decay is discussed in detail in the context of the minimal supersymmetric $S U(5)$. In the case of the minimal SUSY $S U(5)$ we show that if we stick to the strongest bound on the colored triplet mass coming from dimension five proton decay contributions there is no hope to test this model at future nucleon decay experiments through the dimension six operators. We find a lower bound on the partial proton decay lifetime for all relevant channels in the context of flipped SUSY $S U(5)$. We conclude that flipped SUSY $S U(5)$ might be in trouble if proton decay is found at the next generation of experiments with a lifetime below $10^{37}$ years.
\end{abstract}

\section{INTRODUCTION}

The smallest special unitary group that allows embedding of the Standard Model (SM) is $S U(5)$ [1]. On account of its unique symmetry breaking pattern $S U(5)$ represents a fundamental framework to test the idea of grand unification. It is thus necessary to have clear understanding of its generic predictions and testable consequences.

*Electronic address: idorsner@phys.psu.edu

${ }^{\dagger}$ Electronic address: fileviez@cftp.ist.utl.pt

${ }^{\ddagger}$ Electronic address: german.rodrigo@ific.uv.es 
The most dramatic prediction of grand unified theories by far is the decay of the proton [2, 3, 4]. This generic feature has accordingly been a prime target of numerous experimental searches but a positive signal has not yet been observed. Nevertheless, existing experimental limits on the proton decay lifetime have already placed rather severe bounds on simple models of grand unification. This is especially applicable to the minimal models based on $S U(5)$ which will be our preferred framework of interest. We will in particular focus on the current status of these models-in their supersymmetric form-with respect to the latest experimental results on proton decay.

As is well-known non-supersymmetric grand unified theories, in particular the simplest realizations of the ordinary $S U(5)$, were considered for a long time to be ruled out by proton decay. However, it was shown recently that there still exists viable parameter space in minimal realistic models that is yet to be excluded [5, 6]. Also, it has turned out that proton decay might be absent altogether in a class of models based on flipped $S U(5)$ [7]. Both results draw from a recent study that focused on all relevant contributions to proton decay in non-supersymmetric GUTs [8].

The situation with the status of the minimal supersymmetric $S U(5)$ [9] grand unified theory is more involved. This is primarily due to the fact that supersymmetry generates a large number of model dependent proton decay contributions in addition to the non-supersymmetric ones making general analysis intricate. In the context of flipped SUSY $S U(5)$ the dimension six gauge contributions for proton decay are the most important. Therefore, there is no problem to satisfy the current experimental bound on the proton lifetime.

In this work we study the unification constraints in the context of minimal supersymmetric models based on $S U(5)$ and the corresponding implications for proton decay. To be as broad as possible we will look at both types of minimal supersymmetric matter unification under $S U(5)$ - the ordinary and flipped one. In minimal supersymmetric $S U(5)$ we investigate the case when the fields in the adjoint representation $\Sigma_{3}$ (triplet of $S U(2)$ ) and $\Sigma_{8}$ (octet of $S U(3)$ ) are not degenerated. In flipped SUSY SU(5) we study the most general unification constraints. The constraints coming from proton decay are discussed in both models. We find a lower bound on the proton decay lifetime in flipped SUSY $S U(5)$ and conclude that it will be very difficult to test both models, minimal SUSY $S U(5)$ and flipped SUSY $S U(5)$, at future proton decay experiments through the dimension six operators.

Our work is organized as follows: in Section 1 we show the most general unification constraints in minimal supersymmetric $S U(5)$ and their implications for proton decay predictions. In Section 2 we present the relevant predictions in the framework of the minimal supersymmetric version of flipped $S U(5)$. Finally, we summarize our results in Section III. 


\section{MINIMAL SUSY $S U(5)$ : UNIFICATION VERSUS NUCLEON DECAY}

Minimal SUSY $S U(5)$ [9] unifies one generation of matter of the Minimal Supersymmetric Standard Model (MSSM) in two superfields $\hat{\overline{\mathbf{5}}}=\left(\hat{d}^{C}, \hat{L}\right)$ and $\hat{\mathbf{1 0}}=\left(\hat{u}^{C}, \hat{\mathbf{Q}}, \hat{e}^{C}\right)$, while its Higgs sector comprises $\hat{\mathbf{5}}_{\mathbf{H}}=(\hat{T}, \hat{H}), \hat{\overline{\mathbf{5}}}_{\mathbf{H}}=(\hat{\bar{T}}, \hat{\bar{H}})$, and $\hat{\mathbf{2 4}}_{\mathbf{H}}$. In our notation the SM decomposition of the adjoint Higgs superfield reads $\hat{\mathbf{2 4}}=\left(\hat{\Sigma}_{8}, \hat{\Sigma}_{3}, \hat{\Sigma}_{(3,2)}, \hat{\Sigma}_{(\overline{3}, 2)}, \hat{\Sigma}_{24}\right)=(\mathbf{8}, \mathbf{1}, 0)+(\mathbf{1}, \mathbf{3}, 0)+(\mathbf{3}, \mathbf{2},-5 / 6)+(\overline{\mathbf{3}}, \mathbf{2}, 5 / 6)+$ $(\mathbf{1}, \mathbf{1}, 0)$. In addition, it needs to accommodate at least two $S U(5)$ singlet superfields in order to generate observed neutrino masses or use the bilinear R-parity violating interactions.

It has been claimed that this theory, in its renormalizable form and with low-energy SUSY, was excluded on the proton decay grounds [10]. For previous studies see [11, 12, 13]. More precisely, it has been shown [10] that in order to satisfy the experimentally established lower bound on proton lifetime the mass $M_{T}$ of the triplet fields $\hat{T}$ and $\hat{\bar{T}}$ had to be greater than the upper bound on $M_{T}$ that was extracted from requirement to have successful gauge coupling unification. However, it is well-known that the minimal renormalizable SUSY $S U(5)$ is not realistic since it is not possible to establish phenomenologically consistent fermion masses and mixings within its framework. So, the above claim seems redundant to say the least. In fact, any study that aims to rule out any given GUT on the proton decay grounds should be undertaken within a realistic scenario for fermion masses and mixings. We will adhere to this principle in our study.

In order to keep SUSY $S U(5)$ minimal but realistic with respect to the quark and lepton mass spectrum it is sufficient to take into account nonrenormalizable operators in the Yukawa sector [14]. These operators modify the bad relation $Y_{D}=Y_{E}^{T}$ in a way that renders theory realistic. As usual, $Y_{D}\left(Y_{E}\right)$ is the down quark (charged lepton) Yukawa matrix. Once these operators are present the couplings of the triplet Higgs to matter are also modified. In fact, one can even set to zero all the couplings of the triplets to matter [15]. This in turn practically removes any phenomenological bound on the triplet mass. It is thus clear that it is rather difficult to rule out the entire parameter space of the minimal SUSY $S U(5)$. This issue has been studied in detail in reference [16, 17], while the impact of the higher-dimensional operators on proton decay has been studied in [17, 18, 19, 20, 21].

If the higher-dimensional operators are allowed in the Yukawa sector one should for consistency also consider other possible nonrenormalizable operator contributions and investigate their impact on the viability of the theory. We refer to two additional types of operators in particular. The first type modifies the mass spectrum of the Higgs fields responsible for the GUT symmetry breaking with respect to the renormalizable case [17]. The second type affects boundary conditions for the gauge coupling unification through modification of the gauge kinetic terms [22]. It is our intention to investigate in detail influence of the first type 
of operators on the predictions of the theory. As we will show, these modifications alone are sufficient to make theory realistic with respect to the proton decay constraints. This relaxes the need to fine-tune relevant Yukawa couplings to simultaneously recreate observed masses and mixings and suppress couplings of the triplet to matter. Preliminary study of their impact has already been presented in Ref. [17]. Our analysis will not only be more detailed but will also reflect recent improvements in our knowledge of low-energy data as given in Ref. [23]. In addition, we will investigate consequences of particular realizations of the SUSY spectrum on the proton decay predictions. It should finally be mentioned that even the second type of operators is self sufficient in rendering theory realistic with respect to proton decay bounds [4].

\section{A. Unification constraints: octet-triplet splitting}

The mass splitting between octet $\left(\Sigma_{8}\right)$ and triplet $\left(\Sigma_{3}\right)$ of adjoint Higgs superfield as the simplest way to satisfy conservative experimental bound on $M_{T}$ within the minimal SUSY $S U(5)$ framework has first been

suggested in Ref. [17]. The idea is based on the fact that $M_{T}$ scales as $\left(M_{\Sigma_{3}} / M_{\Sigma_{8}}\right)^{5 / 2}$ if successful gauge coupling unification at the one-loop level is imposed. Hence, sufficiently strong $\Sigma_{8}-\Sigma_{3}$ mass splitting could lead to $M_{T}$ being heavy enough to avoid even the most conservative constraints coming from experimental results on partial proton decay lifetimes. However, this approach cannot be implemented in renormalizable theory where $M_{\Sigma_{8}}=M_{\Sigma_{3}}$. To break this degeneracy one needs to consider presence of nonrenormalizable contributions which are anyhow necessary in order to accommodate observed masses of quarks and leptons [14].

To outline how this idea works let us consider the superpotential up to the first order in $\langle\Sigma\rangle / \Lambda$. It reads

$$
W_{\Sigma}=m \operatorname{Tr} \hat{\Sigma}^{2}+\lambda \operatorname{Tr} \hat{\Sigma}^{3}+\frac{\mathrm{a}}{\Lambda}\left(\operatorname{Tr} \hat{\Sigma}^{2}\right)^{2}+\frac{\mathrm{b}}{\Lambda} \operatorname{Tr} \hat{\Sigma}^{4}
$$

where $\langle\Sigma\rangle$ is a vacuum expectation value (VEV) of the order of the GUT scale $\left(M_{\mathrm{GUT}}\right)$ while $\Lambda$ can be identified with the scale of gravity $\left(M_{\text {Planck }}\right)$. If only the first two terms are taken into consideration one obtains $M_{\Sigma_{3}}=M_{\Sigma_{8}}$ if $\langle\Sigma\rangle$ points in the SM direction. This hinders the possibility of increasing $M_{T}$ to arbitrarily high scale since, as we said before, $M_{T}$ depends on $M_{\Sigma_{3}}$ and $M_{\Sigma_{8}}$ only through their ratio. If, on the other hand, one considers a more general scenario-when all the terms in Eq. (1) are taken into account - it is possible to have very wide range of values for the ratio in question. For example, if one neglects for simplicity the term proportional to $\lambda$ in Eq. (1) one obtains $M_{\Sigma_{3}}=4 M_{\Sigma_{8}}$ [17]. That is more than sufficient to bring even the most conservative predictions of the minimal SUSY $S U(5)$ in agreement with experimental findings as we show later.

To explicitly show how $\Sigma_{3}-\Sigma_{8}$ mass splitting enters into prediction for $M_{T}$ we must resort to renormal- 
ization group equations for the SM gauge couplings. At the one-loop level, they are given by:

$$
\begin{aligned}
\alpha_{1}^{-1}\left(M_{Z}\right) & =\alpha_{\mathrm{GUT}}^{-1}+\frac{1}{2 \pi}\left(\frac{41}{10} \ln \frac{M_{\mathrm{GUT}}}{M_{Z}}+\frac{21}{10} \ln \frac{M_{\mathrm{GUT}}}{M_{\tilde{q}}}+\frac{2}{5} \ln \frac{M_{\mathrm{GUT}}}{M_{\tilde{G}}}-10 \ln \frac{M_{\mathrm{GUT}}}{M_{V}}+\frac{2}{5} \ln \frac{M_{\mathrm{GUT}}}{M_{T}}\right), \\
\alpha_{2}^{-1}\left(M_{Z}\right) & =\alpha_{\mathrm{GUT}}^{-1}+\frac{1}{2 \pi}\left(-\frac{19}{6} \ln \frac{M_{\mathrm{GUT}}}{M_{Z}}+\frac{13}{6} \ln \frac{M_{\mathrm{GUT}}}{M_{\tilde{q}}}+2 \ln \frac{M_{\mathrm{GUT}}}{M_{\tilde{G}}}-6 \ln \frac{M_{\mathrm{GUT}}}{M_{V}}+2 \ln \frac{M_{\mathrm{GUT}}}{M_{\Sigma_{3}}}\right), \\
\alpha_{3}^{-1}\left(M_{Z}\right) & =\alpha_{\mathrm{GUT}}^{-1}+ \\
& +\frac{1}{2 \pi}\left(-7 \ln \frac{M_{\mathrm{GUT}}}{M_{Z}}+2 \ln \frac{M_{\mathrm{GUT}}}{M_{\tilde{q}}}+2 \ln \frac{M_{\mathrm{GUT}}}{M_{\tilde{G}}}-4 \ln \frac{M_{\mathrm{GUT}}}{M_{V}}+\ln \frac{M_{\mathrm{GUT}}}{M_{T}}+3 \ln \frac{M_{\mathrm{GUT}}}{M_{\Sigma_{8}}}\right) .
\end{aligned}
$$

Here, for simplicity we assume the same mass $M_{\tilde{q}}$ for all MSSM scalars, i.e., sfermions and the extra Higgs doublet, and the same mass $M_{\tilde{G}}$ for Higgsinos and gauginos. We comment on a more general scenario that accommodates the splitting between the relevant gaugino masses later on. As usual, $M_{V}$ is the mass of superheavy gauge bosons while $M_{G U T}$ represents the scale where gauge couplings unify. We assume $M_{V}=M_{G U T}$ in what follows which is especially justified in the two-loop analysis which we also present towards the end of this section.

It is easy to solve for $M_{T}$ in terms of all other mass scales that appear in Eq. (2). If we eliminate $\alpha_{\mathrm{GUT}}$ we end up with two equations. They read

$$
\begin{aligned}
M_{T} & =\left(\frac{M_{\Sigma_{3}}}{M_{\Sigma_{8}}}\right)^{5 / 2}\left(M_{\tilde{G}}^{4} M_{\tilde{q}} M_{Z}\right)^{1 / 6} \exp \left[-\frac{5}{6} \pi\left(\alpha_{1}^{-1}\left(M_{Z}\right)-3 \alpha_{2}^{-1}\left(M_{Z}\right)+2 \alpha_{3}^{-1}\left(M_{Z}\right)\right)\right], \\
\frac{M_{\Sigma_{3}}}{M_{\Sigma_{8}}} & =\frac{M_{Z}^{22 / 3}}{M_{\Sigma_{8}}^{2} M_{\tilde{G}}^{4 / 3} M_{V}^{4}} \exp \left[\frac{\pi}{3}\left(5 \alpha_{1}^{-1}\left(M_{Z}\right)-3 \alpha_{2}^{-1}\left(M_{Z}\right)-2 \alpha_{3}^{-1}\left(M_{Z}\right)\right)\right] .
\end{aligned}
$$

Of course, $M_{T}$ and $M_{V}$ are then related through

$$
M_{T}=\frac{M_{\tilde{q}}^{1 / 6} M_{Z}^{37 / 2}}{M_{\Sigma_{8}}^{5} M_{\tilde{G}}^{8 / 3} M_{V}^{10}} \exp \left[\frac{10 \pi}{3}\left(\alpha_{1}^{-1}\left(M_{Z}\right)-\alpha_{3}^{-1}\left(M_{Z}\right)\right)\right] .
$$

Clearly, $M_{T}$ scales as $\left(M_{\Sigma_{3}} / M_{\Sigma_{8}}\right)^{5 / 2}$ [17]. Thus, the larger the $M_{\Sigma_{3}} / M_{\Sigma_{8}}$ ratio is the larger $M_{T}$ becomes as we initially suggested. If this ratio is set to one then Eq. (3) simplifies and $M_{T}$ can consequently be easily constrained by low energy input and assumptions with regard to the SUSY spectrum [24]. This in turn implies that the triplet mass is too light to satisfy experimental constraints [10] from proton decay if one neglects the higher dimensional operators and the quark and lepton mixings. In this case we get the strongest bound on the colored triplet mass. So, how much should the ratio depart from one if we want $M_{T}$ to be above the most conservative experimental bound? 
To answer that we first update the result of Ref. [10] according to which the current bound on the partial proton lifetime- $\tau\left(p \rightarrow K^{+} \bar{\nu}\right)>2.3 \times 10^{33}$ years-implies the following bound on the triplet mass: $M_{T}>1.4 \times 10^{17} \mathrm{GeV}$. Using this conservative constraint, $M_{\tilde{G}}=M_{\tilde{q}}=M_{Z}$, and $M_{V}=M_{\mathrm{GUT}}$ we obtain from Eq. (3) the one-loop result

$$
M_{\Sigma_{3}}>2.0 M_{\Sigma_{8}}
$$

So, if one allows for the presence of nonrenormalizable contributions in the superpotential one can certainly make minimal SUSY SU(5) [17] realistic as long as Eq. (6) holds without the need to suppress couplings of the triplet to the matter. In fact, since there are two relevant equations, we obtain an additional constrain. Namely, Eq. (5) simultaneously implies $M_{\Sigma_{8}}<3.0 \times 10^{13} \mathrm{GeV}$ in order that $M_{G U T} \geq M_{T}$.

Let us now study unification constraints on the spectrum of the minimal realistic SUSY $S U(5)$ in detail. Using Eq. (3) and Eq. (4) we can plot the parameter space allowed by unification in the $M_{\Sigma_{3}}-M_{\Sigma_{8}}$ plane for fixed values of $M_{T}$ and $M_{V}=M_{G U T}$. The whole parameter space is shown in Fig. 1 assuming different values of $M_{\tilde{G}}$ and $M_{\tilde{q}}$. The allowed region in the context of the minimal SUSY SU(5) as shown in Fig. 1 is the region bounded from above by $M_{T} \leq M_{V}=M_{\mathrm{GUT}}$, from the left by $M_{V}=M_{\mathrm{GUT}}<M_{\mathrm{Planck}}$ and from the right by $M_{\Sigma_{8}} \leq M_{V}=M_{\mathrm{GUT}}$. The constraint $M_{\Sigma_{3}} \leq M_{V}=M_{\mathrm{GUT}}$ does not play any role due to the other exclusion limits. In Fig. 1h we show the possibility to achieve unification for the case $M_{\tilde{G}}=200 \mathrm{GeV}$ and $M_{\tilde{a}}=1 \mathrm{TeV}$, while in Fig. 1 $\mathrm{b}$ the corresponding parameter space for the so-called "Split SUSY" scenario [25] is shown. To implement relevant experimental bounds on the masses of SUSY particles we use Ref. [23]. Clearly, the allowed region for $M_{\Sigma_{3}}>M_{\Sigma_{8}}$ in Fig. 1b has been considerably reduced with respect to Fig. 11. In all those plots we observe the well known fact that the contributions of the fermionic superpartners are very important for unification, while the contributions of the extra scalars are not relevant at one loop. In order to appreciate this effect notice the differences between Fig. 11 and Fig. 1t where the gaugino mass changes from $200 \mathrm{GeV}$ to $1 \mathrm{TeV}$ and the unification scale for $M_{\Sigma_{3}}=M_{\Sigma_{8}}$ is always above $10^{17} \mathrm{GeV}$ in the latter. In all scenarios shown in Fig. 1 unification scale can be naturally at the string scale [26].

We recall that the triplets could be light once their couplings to matter are suppressed [15]. To stress that point we show the possibility to achieve unification in this case in Fig. 2. It is generated with the same values of input parameters as Fig. 1a but this time we include the region where both $T$ and $\hat{T}$ are very light. That region could be probed at future colliders, particularly at the LHC. For relevant signals at future colliders see reference [27] .

Now, let us study the unification constraints at two-loop level. At the two-loop level, $M_{T}$ picks up dependence on the absolute mass scale of $\Sigma_{8(3)}$. This means that if one considers $M_{\Sigma_{3}}=M_{\Sigma_{8}}$ case and 

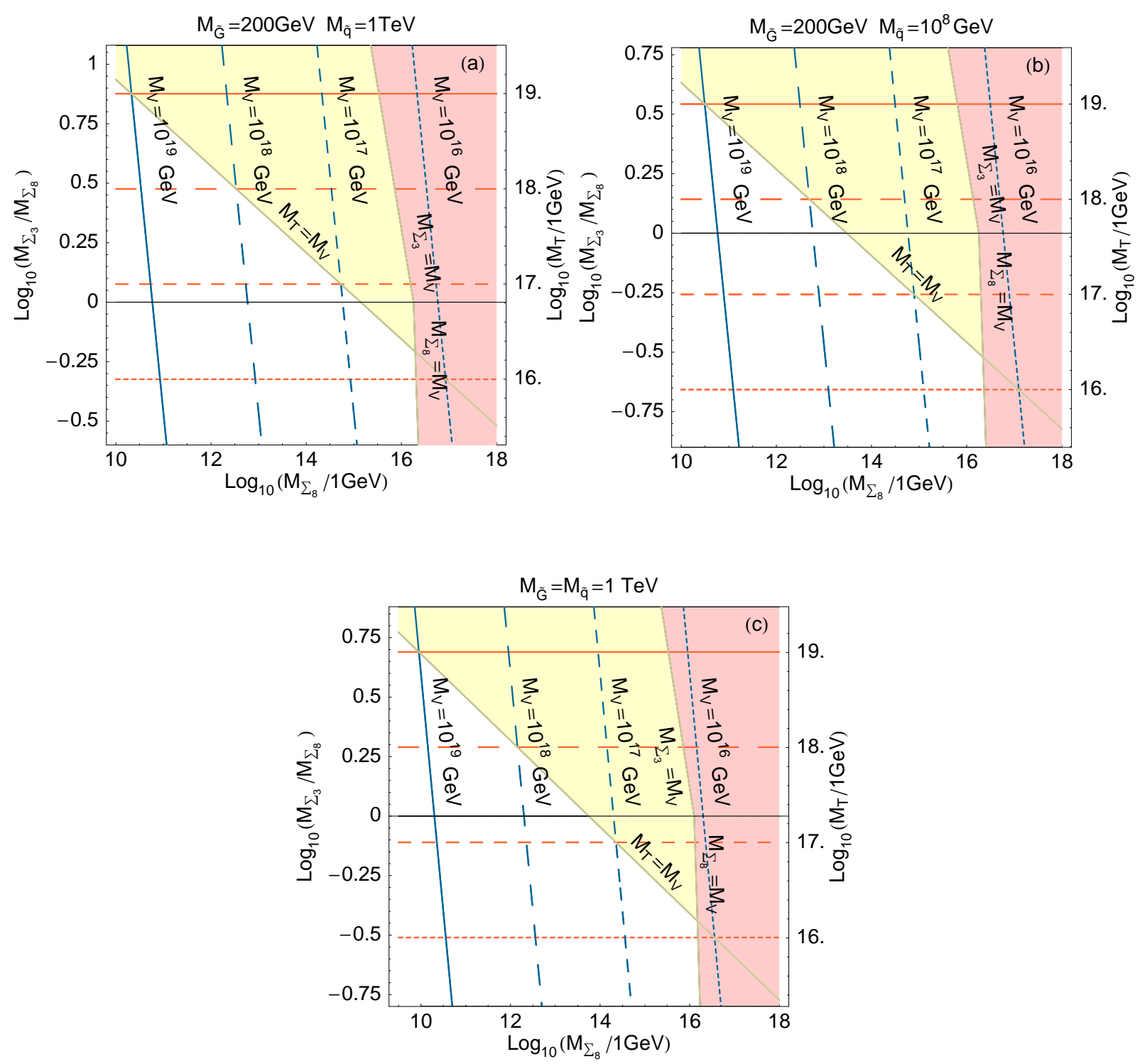

FIG. 1: Parameter space for the gauge coupling unification in the $M_{\Sigma_{3}}-M_{\Sigma_{8}}$ plane for different values of $M_{\tilde{q}}$ and $M_{\tilde{G}}$ in the $\overline{D R}$ scheme. Lines of constant $M_{T}$ and $M_{V}=M_{G U T}$ are shown. The light shaded area is excluded by the constraint $M_{T} \leq M_{V}$ while the dark shaded area is excluded by either $M_{\Sigma_{3}} \leq M_{V}=M_{G U T}$ or $M_{\Sigma_{8}} \leq$ $M_{V}=M_{G U T}$. As input parameters we take $\alpha_{s}\left(M_{Z}\right)_{\overline{M S}}=0.1176, \sin ^{2} \theta_{W}\left(M_{Z}\right)_{\overline{M S}}=0.2312$ and $\alpha\left(M_{Z}\right)_{\overline{M S}}=$ $1 / 127.906$.

allows $M_{\Sigma_{8}}\left(=M_{\Sigma_{3}}\right)$ to be below $M_{T}$ one can establish an upper bound on $M_{T}$ only after one imposes a condition that $M_{G U T}$ is below some natural cutoff. For example, if we take that cutoff to be $M_{\text {Planck }}$, the correct bound for $\tan \beta=4, M_{\tilde{G}}=1 \mathrm{TeV}$ and $M_{\tilde{q}}=1 \mathrm{TeV}$ reads $M_{T}^{0}<1.2 \times 10^{16} \mathrm{GeV}$. Here, $M_{T}^{0}$ 


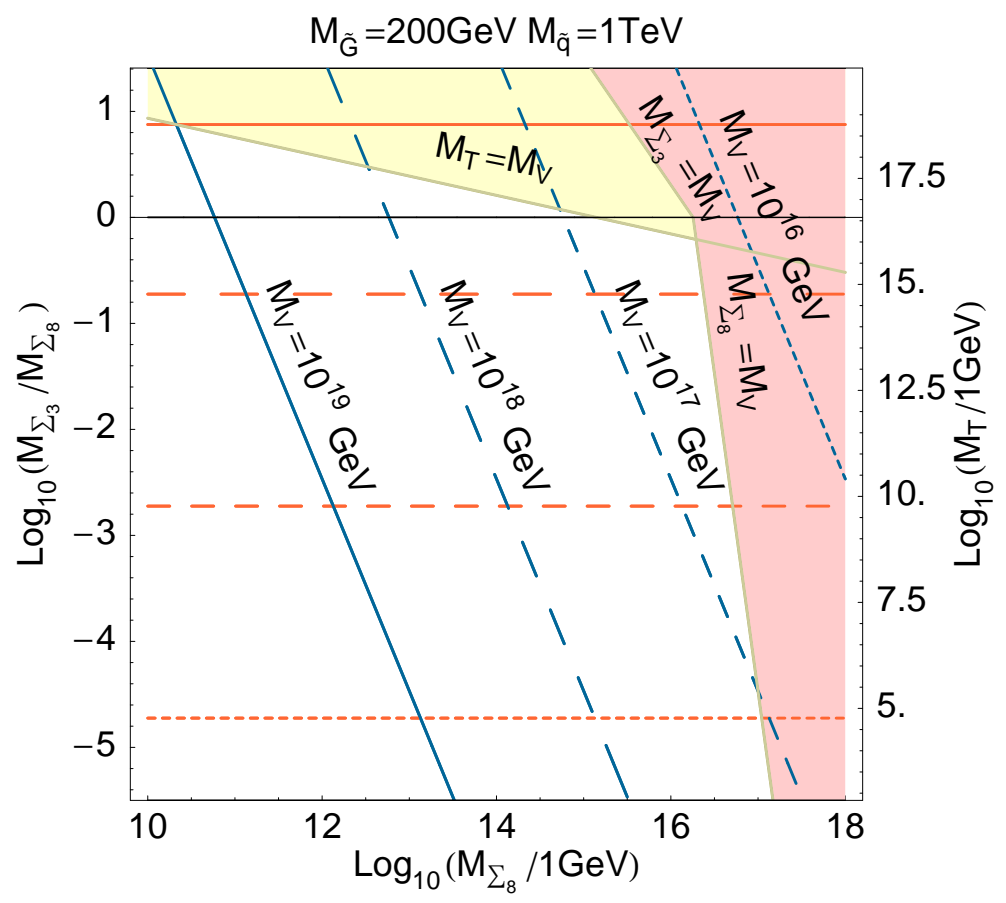

FIG. 2: The whole parameter space for gauge coupling unification for light $M_{T}$ scenario in the $M_{\Sigma_{3}}-M_{\Sigma_{8}}$ plane. Input parameters are the same as in Fig. 19.

represents the mass of the triplet for the case when $M_{\Sigma_{3}}=M_{\Sigma_{8}}$. In fact, we find

$$
5.13_{-2.63}^{+5.07} \times 10^{15} \mathrm{GeV} \leq M_{T}^{0} \leq 1.2_{-0.61}^{+1.18} \times 10^{16} \mathrm{GeV},
$$

in order for successful unification to take place. This range of values for $M_{T}^{0}$ is obtained by allowing for arbitrary absolute mass scale of degenerate $\Sigma_{3}$ and $\Sigma_{8}$ fields. For the values quoted in Eq. (7) this scale varies between $7.3 \times 10^{10} \mathrm{GeV}$ for the upper bound and $1.5 \times 10^{16} \mathrm{GeV}$ for the lower bound, respectively. Obviously, the constraint on $M_{T}^{0}$ is rather tight. Quoted uncertainties in Eq. (7) reflect $1 \sigma$ uncertainty in $\alpha_{s}\left(M_{Z}\right)_{\overline{M S}}=0.1176 \pm 0.0020$ as given in [23]. We stress again that the upper bound is cutoff dependent.

If we depart from the $M_{\Sigma_{3}}=M_{\Sigma_{8}}$ assumption then the upper limit on $M_{T}$ basically corresponds to the cutoff of the theory. We opt to present the two-loop analysis in Fig. 3 as a contour plot of the masses of $\Sigma_{8}$ and Higgs triplet fields in the $M_{G U T}-M_{\Sigma_{3}} / M_{\Sigma_{8}}$ plane. In order to generate it we use values for gauge couplings at $M_{Z}$ taken from Ref. [23]. In addition, we take into account CKM mixing parameters as given in Ref. [23] and include the effect of all three families. We consider only the $\tan \beta=4$ case. In our analysis the exact numerical solutions is generated for sufficient number of points to have smooth interpolation.

Some comments are in order with respect to Fig. 3 Vertical errors on the points that give $M_{T}=$ 
$1.4 \times 10^{17} \mathrm{GeV}$ line correspond to the case when $\alpha_{s}\left(M_{Z}\right)_{\overline{M S}}$ is varied within $1 \sigma$ while $M_{G U T}$ and $M_{T}$ are kept fixed. In other words, what is varied there are the ratio $M_{\Sigma_{3}} / M_{\Sigma_{8}}$ and $M_{\Sigma_{8}}$. Horizontal errors on one of the points on the $M_{T}=1.4 \times 10^{17} \mathrm{GeV}$ line also correspond to the $1 \sigma$ variation in $\alpha_{s}\left(M_{Z}\right) \overline{M S}$. This time $M_{\Sigma_{8}}$ and $M_{G U T}$ are varied with $M_{\Sigma_{3}} / M_{\Sigma_{8}}$ and $M_{T}$ held fixed. Both types of error bars are given to demonstrate the impact of the least experimentally known input parameter. The dependence on $\tan \beta$ is practically negligible.

The only other major dependence of $M_{T}$ is on the exact spectrum of the SUSY particles here encoded in parameters $M_{\tilde{G}}$ and $M_{\tilde{q}}$ for simplicity. This dependence can be treated in a satisfactory manner only if and when this spectrum is experimentally establish and/or better constrained. In any case, if we assume $M_{\tilde{G}}=M_{\tilde{q}}=500 \mathrm{GeV}$ instead of $M_{\tilde{G}}=M_{\tilde{q}}=1 \mathrm{TeV}$ then $M_{T}=1.4 \times 10^{17} \mathrm{GeV}$ line in Fig. [3 is given by the thick line. As one can see, the "overall" change in the SUSY scale within the region that can be directly probed in experiments is still less significant than the uncertainty in $\alpha_{s}$.

There is however one important point that regards exact SUSY spectrum that we need to address. Namely, it is well known that the GUT scale unification of gaugino masses implies that $M_{1} / \alpha_{1}=M_{2} / \alpha_{2}=$ $M_{3} / \alpha_{3}$ at any given scale up to small corrections. Here $M_{1}, M_{2}$ and $M_{3}$ are the Bino, Wino and gluino masses, respectively. We can thus infer that at low-scale gaugino unification predicts $M_{3} / M_{2} \simeq 3.5$. This, on the other hand, is obviously in conflict with our assumption of gaugino degeneracy. It is a simple exercise to show that, at the one-loop level, $M_{T}$ scales as $\left(M_{2} / M_{3}\right)^{5 / 3}$. So, $M_{T}$ dependence on the ratio of relevant gaugino masses is less severe than on the $M_{\Sigma_{3}}-M_{\Sigma_{8}}$ mass splitting. In other words, we have captured the dominant effect that controls predictions for $M_{T}$ within the minimal framework. But, if one assumes that this pattern of gaugino masses is indeed the correct one it is easy to apply it to our analysis. For example, the limits that are quoted in Eq. (7) should be divided by 8. Only then our results could be compared with the existing results in the literature such as the one in Ref. [10].

Fig. 3 covers the parameter space of the minimal SUSY $S U(5)$ in both renormalizable and nonrenormalizable case and it extends only to $M_{G U T}<2 \times 10^{18} \mathrm{GeV}$. We show the explicit dependence of $M_{T}$ on all relevant parameters, including $M_{G U T}$ and absolute scale of $M_{\Sigma_{8}}$. Gray box in Fig. 3 marks a point at which $M_{\Sigma_{3}}=M_{T}=M_{G U T}$, while empty box represents a point where $M_{\Sigma_{3}}=M_{\Sigma_{8}}=M_{G U T}$. Note that the error bars at the latter point correspond to the $1 \sigma$ variation of $\alpha_{s}$ as shown on the left side of Eq. (7). The error bars are much smaller there with respect to error bars elsewhere since only $M_{T}^{0}$ is allowed to vary to generate successful unification.

Last point we want to discuss is the fact that more recent lattice QCD evaluations [28, 29, 30] of the proton decay matrix element consistently imply a value that is larger than the value $\left(\alpha_{H}=0.003 \mathrm{GeV}^{3}\right)$ used to derive the limit $M_{T}>1.4 \times 10^{17} \mathrm{GeV}$. Ref. [10] offers alternative, even more stringent experimental 


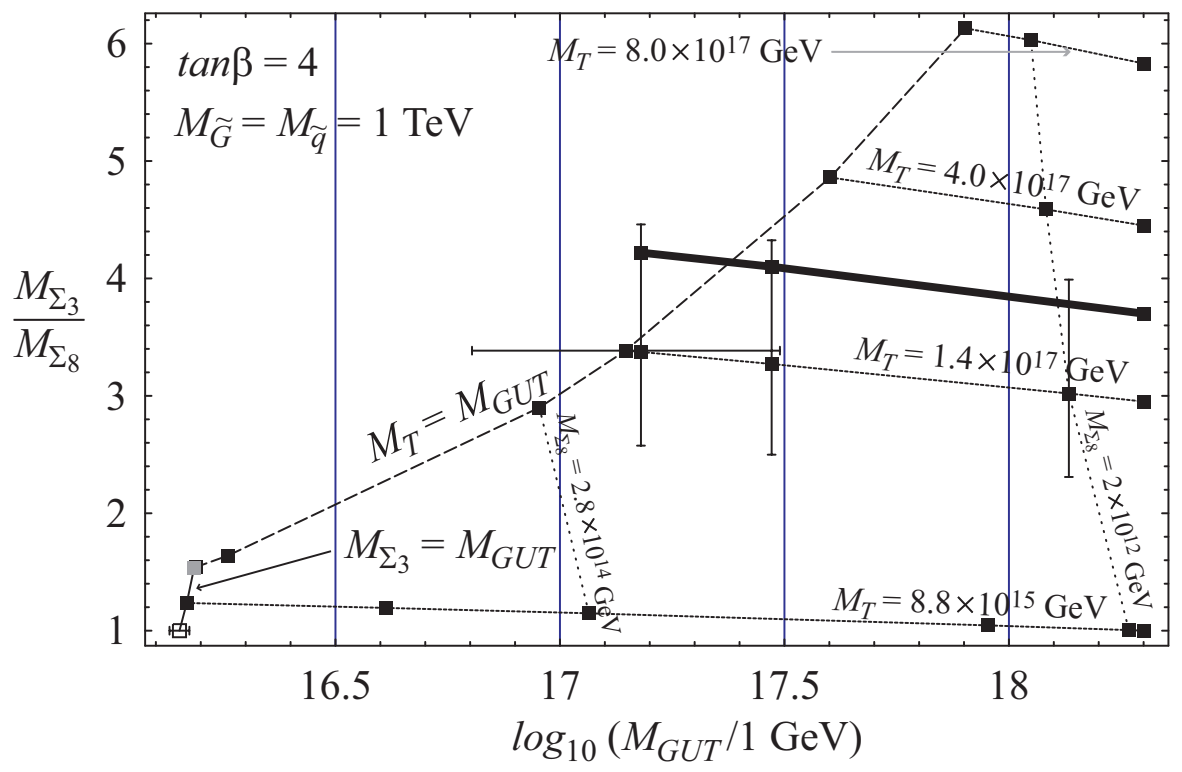

FIG. 3: Parameter space for successful gauge coupling unification at the two-loop level in the $M_{G U T^{-}}-M_{\Sigma_{3}} / M_{\Sigma_{8}}$ plane. Lines of constant $M_{T}$ and $M_{\Sigma_{8}}$ are shown. The dashed line corresponds to $M_{T}=M_{G U T}$. Points (boxes) are exact numerical solutions for central value of input parameters while lines represent appropriate linear interpolation. Input parameters are specified in detail in the text as well as the origin of shown error bars.

limit on $M_{T}$, based on the value $\alpha_{H}=0.014 \mathrm{GeV}^{3}$ [28]. When we update it to incorporate the latest experimental bound on the partial proton lifetime it reads $M_{T}>3.7 \times 10^{17} \mathrm{GeV}$. One can see from Fig. 3 that even this limit can be satisfied with $M_{\Sigma_{3}} / M_{\Sigma_{8}} \geq 4$. As far as the correctness of the Eq. (6) is concerned, at the two-loop level, for the central values of our set of input parameters, it should read

$$
M_{\Sigma_{3}}>2.6 M_{\Sigma_{8}}
$$

in order to have $M_{T}>1.4 \times 10^{17} \mathrm{GeV}$.

\section{B. $\quad d=6$ proton decay in SUSY $S U(5)$}

Let us investigate the predictions for proton decay. Since the $d=6$ contributions are the least model dependent we focus on them. Assuming $Y_{U}=Y_{U}^{T}$ [31] the decay rates due to the presence of the superheavy gauge bosons are:

$$
\begin{gathered}
\Gamma\left(p \rightarrow \pi^{+} \bar{\nu}\right)=\frac{\pi m_{p} \alpha_{G U T}^{2}}{2 f_{\pi}^{2} M_{G U T}^{4}} A_{L}^{2}\left|\alpha_{H}\right|^{2}(1+D+F)^{2}\left|V_{C K M}^{11}\right|^{2}, \\
\Gamma\left(p \rightarrow K^{+} \bar{\nu}\right)=\frac{\pi \alpha_{G U T}^{2}}{2 f_{\pi}^{2} M_{G U T}^{4}} \frac{\left(m_{p}^{2}-m_{K}^{2}\right)^{2}}{m_{p}^{3}} A_{L}^{2}\left|\alpha_{H}\right|^{2}\left[A_{1}^{2}\left|V_{C K M}^{11}\right|^{2}+A_{2}^{2}\left|V_{C K M}^{12}\right|^{2}\right]^{2},
\end{gathered}
$$


where

$$
\begin{aligned}
& A_{1}=\frac{2}{3} \frac{m_{p}}{m_{B}} D \\
& A_{2}=1+\frac{1}{3} \frac{m_{p}}{m_{B}}(D+3 F) .
\end{aligned}
$$

In the above equations $m_{B}$ is the average baryon mass. $D, F$ and $\alpha_{H}$ are the parameters of the chiral Lagrangian, while $A_{L}$ takes into account the renormalization effects. See reference [4] for details.

Let us study the impact of the unification constraints studied before on the proton lifetime. We have noticed that in all scenarios showed in Fig. 1 the unification scale is always $M_{G U T} \gtrsim 10^{16} \mathrm{GeV}$, therefore we can set a lower bound on the partial proton lifetimes:

$$
\begin{aligned}
\tau\left(p \rightarrow \pi^{+} \bar{\nu}\right) & \gtrsim 8 \times 10^{35} \text { years }, \\
\tau\left(p \rightarrow K^{+} \bar{\nu}\right) & \gtrsim 7.6 \times 10^{37} \text { years } .
\end{aligned}
$$

Here we have used $\alpha_{H}=0.015 \mathrm{GeV}^{3}[29$, 30]. Notice that those lower bounds are very conservative, and valid for a minimal realistic supersymmetric $S U(5)$ with $Y_{U}=Y_{U}^{T}$. The values above tell us that if we want to test the predictions coming from $d=6$ operators in minimal supersymmetric $S U(5)$ in the next generation of proton decay experiments the lower bounds have to be improved by at least four orders of magnitude. For new proposals of proton decay experiments see Ref. [32].

Now, let us discuss the correlation between the unification constraints, and the predictions for nucleon decay coming from the $d=5$ and $d=6$ contributions. We have argued that in the realistic SUSY $S U(5)$ there is no a well-defined lower bound on $M_{T}$. However, if we stick to the strongest bound on $M_{T}, M_{T}>$ $10^{17} \mathrm{GeV}$, coming from $d=5$ proton decay we can conclude that in this case the mass of the superheavy gauge bosons is always, $M_{V}>10^{17} \mathrm{GeV}$ and the partial proton decay lifetimes read $\tau\left(p \rightarrow \pi^{+} \bar{\nu}\right) \gtrsim$ $8 \times 10^{39}$ years, and $\tau\left(p \rightarrow K^{+} \bar{\nu}\right) \gtrsim 7.6 \times 10^{41}$ years. In this case there is no hope to test the minimal supersymmetric $S U(5)$ at future proton decay experiments.

\section{MINIMAL SUSY FLIPPED $S U(5)$ : UNIFICATION VERSUS NUCLEON DECAY}

There exists another possibility to use $S U(5)$ to partially unify the SM matter fields. In that approach the electric charge which is a generator of the conventional $S U(5)$ is taken to be a linear combination of generators operating in both $S U(5)$ and an extra $U(1)$. This approach leads to a so-called flipped $S U(5)$ [33, 34, 35, 36]. The matter unifies but its embedding in $S U(5)$ differs with respect to the ordinary $S U(5)$ assignment; it can be obtained by the following flip: $d^{C} \leftrightarrow u^{C}, e^{C} \leftrightarrow \nu^{C}, u \leftrightarrow d$ and $\nu \leftrightarrow e$. Since the matter unification differs from what one has in ordinary $S U(5)$, the proton decay predictions are also 
different [34]. In what follows we will investigate unification constraints on the mass spectrum of the minimal flipped $S U(5)$ and corresponding implications for proton decay signatures. For recent studies in this context see reference [10, 37]. However, in those previous studies the authors did not studied in detail the possibility to test this model at future proton decay experiments.

Unlike ordinary $S U(5)$ the minimal flipped $S U(5)$ does not require adjoint Higgs but $\mathbf{1 0}$ and $\overline{\mathbf{1 0}}$ of Higgs to break down to the SM. These two representations economically implement the so-called "missing partner mechanism" that efficiently suppresses $d=5$ proton decay operators [36]. Due to that the dominant contribution to proton decay amplitudes comes from the gauge boson exchange. Hence, the only relevant scale for proton decay is set by their mass $M_{V^{\prime}}$.

In order to constrain $M_{V^{\prime}}$ we use gauge coupling unification. Since only $S U(2)$ and $S U(3)$ are fully embedded in $S U(5)$ we accordingly require unification of $\alpha_{2}$ and $\alpha_{3}$ only. The relevant equations are

$$
\begin{aligned}
\alpha_{2}^{-1}\left(M_{Z}\right) & =\alpha_{\mathrm{GUT}}^{-1}+\frac{1}{2 \pi}\left(-\frac{19}{6} \ln \frac{M_{\mathrm{GUT}}}{M_{Z}}+\frac{13}{6} \ln \frac{M_{\mathrm{GUT}}}{M_{\tilde{q}}}+2 \ln \frac{M_{\mathrm{GUT}}}{M_{\tilde{G}}}-6 \ln \frac{M_{\mathrm{GUT}}}{M_{V^{\prime}}}\right), \\
\alpha_{3}^{-1}\left(M_{Z}\right) & =\alpha_{\mathrm{GUT}}^{-1}+ \\
& +\frac{1}{2 \pi}\left(-7 \ln \frac{M_{\mathrm{GUT}}}{M_{Z}}+2 \ln \frac{M_{\mathrm{GUT}}}{M_{\tilde{q}}}+2 \ln \frac{M_{\mathrm{GUT}}}{M_{\tilde{G}}}-4 \ln \frac{M_{\mathrm{GUT}}}{M_{V^{\prime}}}+2 \ln \frac{M_{\mathrm{GUT}}}{M_{T^{\prime}}}\right),
\end{aligned}
$$

where $M_{T^{\prime}}$ is a common mass for the triplet Higgs fields. The number of triplets in flipped $S U(5)$ is twice the number of triplets in ordinary $S U(5)$. That is the reason why our Eqs. (14) defer from what has been presented in Ref. [10]. In any case, the solution to these equations is

$$
M_{T^{\prime}}=\frac{M_{Z}^{23 / 12} M_{\tilde{q}}^{1 / 12}}{M_{V^{\prime}}} \exp \left[\pi\left(\alpha_{2}^{-1}\left(M_{Z}\right)-\alpha_{3}^{-1}\left(M_{Z}\right)\right)\right] .
$$

Again, we consider the case $M_{V^{\prime}}=M_{G U T}$, where $M_{G U T}$ represents the scale where $\alpha_{2}$ and $\alpha_{3}$ unify. Note that Eq. (15) is valid as long as $M_{T^{\prime}} \leq M_{G U T}$. This immediately yields

$$
M_{V^{\prime}} \equiv M_{G U T} \geq M_{Z} \exp \left[\frac{\pi}{2}\left(\alpha_{2}^{-1}\left(M_{Z}\right)-\alpha_{3}^{-1}\left(M_{Z}\right)\right)\right]
$$

where we have used $M_{\tilde{q}} \geq M_{Z}$. Notice that the dependence on $M_{\tilde{q}}$ is extremely weak. The right-hand side of Eq. (16) contains only the low-energy input which is well-known. Upon inserting the latest experimental values we obtain the lower bound $M_{V^{\prime}} \geq 2.34 \times 10^{16} \mathrm{GeV}$. This bound allows us to set a lower bound on proton lifetime as we show later.

The available parameter space in the minimal flipped $S U(5)$ for the exact unification of $\alpha_{2}$ and $\alpha_{3}$ in the $M_{V^{\prime}}-M_{T^{\prime}}$ plane is shown in Fig. 4, The allowed region is bounded by the constraints $M_{Z} \leq M_{\tilde{q}} \leq$ $10^{12} \mathrm{GeV}$, and $M_{T^{\prime}} \leq M_{V^{\prime}}$. The upper bound on $M_{\tilde{q}}$ is coming from the cosmological limit on the gluino 


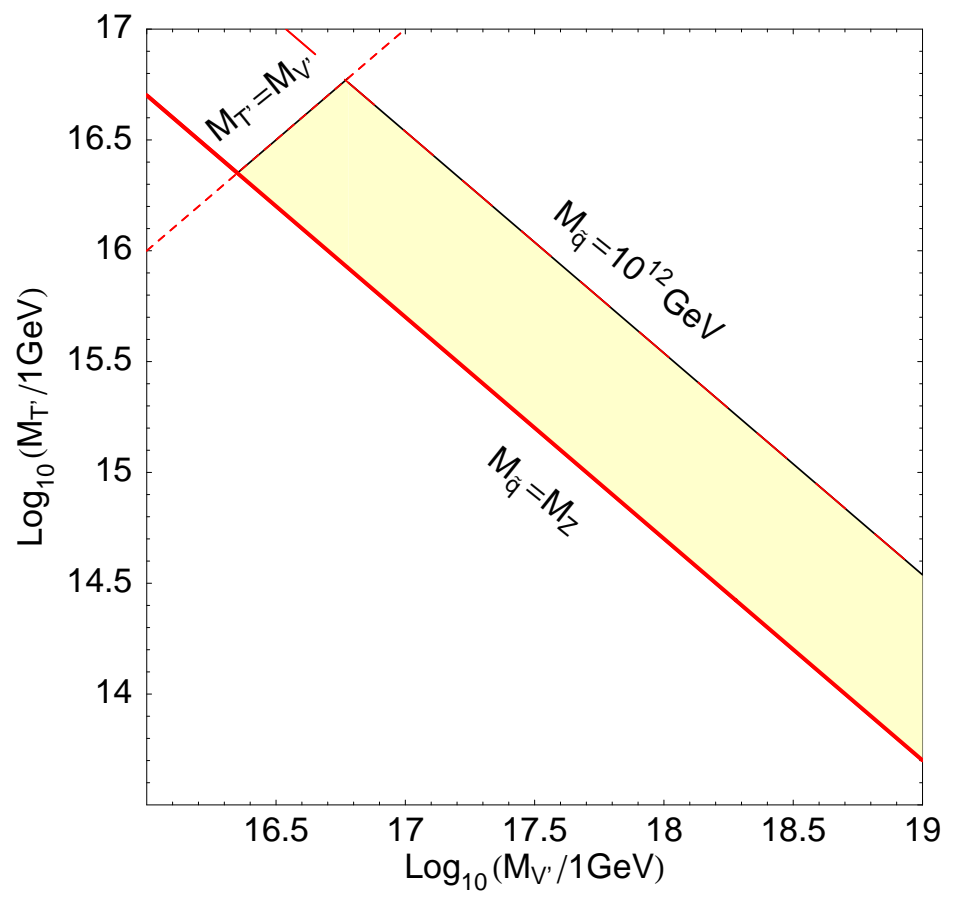

FIG. 4: Viable parameter space (shaded region) of the minimal flipped SUSY $S U(5)$ in the $M_{V^{\prime}}-M_{T^{\prime}}$ plane in the $\overline{D R}$ scheme. Same input values as in Fig. 1.

lifetime [38]. Notice that as in the case of minimal SUSY SU(5) the unification of $\alpha_{2}$ and $\alpha_{3}$ could be at the string scale.

Finally, let us incorporate the GUT scale unification of gaugino masses in the minimal flipped $S U(5)$ context. This time it implies $M_{2} / \alpha_{2}=M_{3} / \alpha_{3}$ at any given scale up to small corrections. Simple exercise yields

$$
M_{G U T} \geq M_{Z}^{23 / 24} M_{\tilde{q}}^{1 / 24}\left(\frac{M_{\tilde{H}}}{M_{3}}\right)^{1 / 6}\left(\frac{M_{2}}{M_{3}}\right)^{1 / 3} \exp \left[\frac{\pi}{2}\left(\alpha_{2}^{-1}\left(M_{Z}\right)-\alpha_{3}^{-1}\left(M_{Z}\right)\right)\right],
$$

where $M_{\tilde{H}}$ stands for the Higgsino masses. The dependence on the ratio of relevant gaugino masses is much weaker than in the ordinary $S U(5)$. And, unlike in ordinary $S U(5), M_{T}$ does depend on the absolute scales of $M_{2}$ and $M_{3}$. The heavier $M_{3}$ is the lighter $M_{G U T}$ becomes although this dependence is also very weak. It is thus easy to see that possible gaugino unification has basically no impact on the $M_{V^{\prime}}$ bound we quoted earlier. 


\section{A. $d=6$ proton decay in flipped SUSY $S U(5)$}

Let us now turn our attention to proton decay signatures. In Ref. [39] it has been shown the possibility to make clean tests of minimal flippled $S U(5)$ through the channel

$$
\Gamma\left(p \rightarrow \pi^{+} \bar{\nu}\right)=k^{4} C_{2}
$$

and the ratio between different channels with charged antileptons

$$
\frac{\Gamma\left(p \rightarrow K^{0} e^{+}\right)}{\Gamma\left(p \rightarrow \pi^{0} e^{+}\right)}=\frac{\Gamma\left(p \rightarrow K^{0} \mu^{+}\right)}{\Gamma\left(p \rightarrow \pi^{0} \mu^{+}\right)}=2 \frac{C_{3}}{C_{2}} \frac{\left|V_{C K M}^{12}\right|^{2}}{\left|V_{C K M}^{11}\right|^{2}}=0.018
$$

where $k=g_{5} / \sqrt{2} M_{V^{\prime}}^{-1}$, and

$$
\begin{aligned}
& C_{2}=m_{p} A_{L}^{2}\left|\alpha_{H}\right|^{2}(1+D+F)^{2} / 8 \pi f_{\pi}^{2}, \\
& C_{3}=\frac{\left(m_{p}^{2}-m_{K}^{2}\right)^{2}}{8 \pi f_{\pi}^{2} m_{p}^{3}} A_{L}^{2}\left|\alpha_{H}\right|^{2}\left[1+\frac{m_{p}}{m_{B}}(D-F)\right]^{2} .
\end{aligned}
$$

Recall that the Yukawa matrix for down quarks is symmetric in minimal flipped $S U(5)$, and $\Gamma(p \rightarrow$ $\left.K^{+} \bar{\nu}\right)=\Gamma\left(n \rightarrow K^{0} \bar{\nu}\right)=0$. See reference [4] for details and the values of the different constants in the equations above.

To set a bound on the partial proton lifetime we need the value of $\alpha_{G U T}\left(=g_{5}^{2} /(4 \pi)\right)$ that corresponds to the minimal GUT scale. This can be obtained from Eq. (14):

$$
\alpha_{\mathrm{GUT}}^{-1}=\alpha_{2}^{-1}\left(M_{Z}\right)-\frac{1}{2 \pi} \ln \frac{M_{\mathrm{GUT}}}{M_{Z}} .
$$

Taking into account our lower bound on $M_{\mathrm{GUT}}$ we obtain $\alpha_{\mathrm{GUT}}^{-1} \leq 24.24$. Now, using $\alpha_{H}=0.015 \mathrm{GeV}^{3}$ the lower bound on the partial proton lifetime is

$$
\tau\left(p \rightarrow \pi^{+} \bar{\nu}\right) \geq 2.15 \times 10^{37} \text { years }
$$

Using this lower bound we can obtain a lower bound on the partial proton lifetime for the channels with charged antileptons [39]:

$$
\tau\left(p \rightarrow \pi^{0} e^{+}\left(\mu^{+}\right)\right)>2 \tau\left(p \rightarrow \pi^{+} \bar{\nu}\right)
$$

As in the previous section, we conclude that it will be very difficult to test the minimal flipped $S U(5)$ at the next generation of proton decay experiments by looking at the channel $p \rightarrow \pi^{+} \bar{\nu}$ and using the ratio given in Eq. (19). We recall that in the near future the lower bounds will be improved by two or three orders of magnitude. If proton decay is found, in particular the channels above, and the lifetime is below this lower bound then minimal flipped SUSY $S U(5)$ will be highly disfavored. 


\section{CONCLUSIONS}

We have investigated the unification constraints in the minimal supersymmetric grand unified theories based on $S U(5)$. The most general constraints on the spectrum of minimal supersymmetric $S U(5)$ and flipped $S U(5)$ have been shown. The upper bound on the mass of the colored Higgs mediating proton decay has been discussed in detail. We studied the issue of proton decay in both GUT models, pointing out lower bounds on the partial proton lifetime for the relevant channels in flipped SUSY $S U(5)$. We conclude that if proton decay is found in the next generation of experiments with a lifetime lower than $10^{37}$ years, then flipped $S U(5)$ could be excluded as a realistic GUT candidate. In the case of the minimal supersymmetric $S U(5)$ we have shown that if we stick to the strongest bound on the colored triplet mass coming from dimension five proton decay contributions there is no hope to test the model at future nucleon decay experiments through the dimension six operators.

\section{Acknowledgements}

The work of G. R. was partially supported by Ministerio de Educación y Ciencia (MEC) under grant FPA200400996, Generalitat Valenciana under grant GV05-015, Consejo Superior de Investigaciones Científicas (CSIC) under grant PIE 200650I247, and European Commission FLAVIAnet MRTN-CT-2006-035482. P. F. P. has been supported by Fundação para a Ciência e a Tecnologia (FCT, Portugal) through the project CFTP,POCTI-SFA-2-777 and a fellowship under project POCTI/FNU/44409/2002. P. F. P would like to thank the Instituto de Física Corpuscular (IFIC) in Valencia for hospitality and B. Bajc and G. Senjanović for discussions.

[1] H. Georgi and S. L. Glashow, “Unity Of All Elementary Particle Forces,” Phys. Rev. Lett. 32 (1974) 438.

[2] J. C. Pati and A. Salam, "Is Baryon Number Conserved?," Phys. Rev. Lett. 31 (1973) 661.

[3] P. Langacker, “Grand Unified Theories And Proton Decay,” Phys. Rept. 72 (1981) 185.

[4] P. Nath and P. Fileviez Pérez, "Proton stability in grand unified theories, in strings, and in branes," arXiv:hep-ph/0601023.

[5] I. Dorsner and P. Fileviez Pérez, "Unification without supersymmetry: Neutrino mass, proton decay and light leptoquarks," Nucl. Phys. B 723 (2005) 53 [arXiv:hep-ph/0504276]; I. Dorsner, P. Fileviez Pérez and R. Gonzalez Felipe, "Phenomenological and cosmological aspects of a minimal GUT scenario," Nucl. Phys. B 747 (2006) 312 [arXiv:hep-ph/0512068].

[6] I. Dorsner, P. Fileviez Pérez and G. Rodrigo, "Fermion masses and the UV cutoff of the minimal realistic SU(5)," arXiv:hep-ph/0607208 
[7] I. Dorsner and P. Fileviez Pérez, "Could we rotate proton decay away?," Phys. Lett. B 606 (2005) 367 [arXiv:hep-ph/0409190].

[8] I. Dorsner and P. Fileviez Pérez, "How long could we live?," Phys. Lett. B 625 (2005) 88 [arXiv:hep-ph/0410198].

[9] S. Dimopoulos and H. Georgi, "Softly Broken Supersymmetry And SU(5)," Nucl. Phys. B 193 (1981) 150; N. Sakai, “Naturalness In Supersymmetric 'Guts'," Z. Phys. C 11 (1981) 153.

[10] H. Murayama and A. Pierce, "Not even decoupling can save minimal supersymmetric SU(5)," Phys. Rev. D 65 (2002) 055009 [arXiv:hep-ph/0108104].

[11] P. Nath, A. H. Chamseddine and R. Arnowitt, “Nucleon Decay In Supergravity Unified Theories," Phys. Rev. D 32 (1985) 2348.

[12] R. Arnowitt, A. H. Chamseddine and P. Nath, "Nucleon Decay Branching Ratios In Supergravity SU(5) Guts," Phys. Lett. B 156 (1985) 215.

[13] J. Hisano, H. Murayama and T. Yanagida, "Nucleon decay in the minimal supersymmetric SU(5) grand unification,” Nucl. Phys. B 402 (1993) 46 [arXiv:hep-ph/9207279].

[14] J. R. Ellis and M. K. Gaillard, “Fermion Masses And Higgs Representations In SU(5),” Phys. Lett. B 88 (1979) 315.

[15] G. R. Dvali, “Can 'doublet - triplet splitting' problem be solved without doublet - triplet splitting?," Phys. Lett. B 287 (1992) 101.

[16] B. Bajc, P. Fileviez Pérez and G. Senjanović, “Proton decay in minimal supersymmetric SU(5)," Phys. Rev. D 66 (2002) 075005 [arXiv:hep-ph/0204311].

[17] B. Bajc, P. Fileviez Pérez and G. Senjanović, "Minimal supersymmetric SU(5) theory and proton decay: Where do we stand?," arXiv:hep-ph/0210374, P. Fileviez Pérez, "Phenomenological aspects of supersymmetric gauge theories," arXiv:hep-ph/0310199.

[18] P. Nath, "Hierarchies and Textures in Supergravity Unification," Phys. Rev. Lett. 76 (1996) 2218 [arXiv:hep-ph/9512415].

[19] P. Nath, "Textured Minimal and Extended Supergravity Unification and Implications for Proton Stability," Phys. Lett. B 381 (1996) 147 [arXiv:hep-ph/9602337].

[20] Z. Berezhiani, Z. Tavartkiladze and M. Vysotsky, "d = 5 operators in SUSY GUT: Fermion masses versus proton decay," arXiv:hep-ph/9809301

[21] D. Emmanuel-Costa and S. Wiesenfeldt, "Proton decay in a consistent supersymmetric SU(5) GUT model," Nucl. Phys. B 661 (2003) 62 [arXiv:hep-ph/0302272].

[22] C. T. Hill, “Are There Significant Gravitational Corrections To The Unification Scale?," Phys. Lett. B 135 (1984) 47. Q. Shafi and C. Wetterich, "Modification Of GUT Predictions In The Presence Of Spontaneous Compactification,” Phys. Rev. Lett. 52 (1984) 875.

[23] W. M. Yao et al. [Particle Data Group], "Review of particle physics,” J. Phys. G 33 (2006) 1.

[24] R. Barbieri and L. J. Hall, "Grand unification and the supersymmetric threshold,” Phys. Rev. Lett. 68 (1992) 752; J. Hisano, H. Murayama and T. Yanagida, "Probing GUT scale mass spectrum through precision measurements 
on the weak scale parameters," Phys. Rev. Lett. 69 (1992) 1014.

[25] N. Arkani-Hamed and S. Dimopoulos, "Supersymmetric unification without low energy supersymmetry and signaturesfor fine-tuning at the LHC," JHEP 0506 (2005) 073 [arXiv:hep-th/0405159]; G. F. Giudice and A. Romanino, "Split supersymmetry," Nucl. Phys. B 699 (2004) 65 [Erratum-ibid. B 706 (2005) 65] [arXiv:hep-ph/0406088].

[26] C. Bachas, C. Fabre and T. Yanagida, "Natural gauge-coupling unification at the string scale," Phys. Lett. B 370 (1996) 49 [arXiv:hep-th/9510094].

[27] K. Cheung and G. C. Cho, "TeV colored Higgsinos in alternative grand unified theories," Phys. Rev. D 69 (2004) 017702 [arXiv:hep-ph/0306068].

[28] Y. Kuramashi [JLQCD Collaboration], "Nucleon decay matrix elements from lattice QCD," arXiv:hep-ph/0103264

[29] Y. Aoki [RBC Collaboration], "Nucleon decay matrix elements with N(f) $=0$ and 2 domain-wall quarks," Nucl. Phys. Proc. Suppl. 140 (2005) 405 [arXiv:hep-lat/0409114].

[30] Y. Aoki, C. Dawson, J. Noaki and A. Soni, "Proton decay matrix elements with domain-wall fermions," arXiv:hep-lat/0607002

[31] P. Fileviez Pérez, "Fermion mixings vs $d=6$ proton decay," Phys. Lett. B 595 (2004) 476 [arXiv:hep-ph/0403286].

[32] Talks given by S. Katsanevas, K. Nakamura, R. Wilson and A. de Bellefon at Workshop on Next generation Nucleon decay and Neutrino detectors 2006 (NNN06), http://neutrino.phys.washington.edu/nnn06/

[33] A. De Rujula, H. Georgi and S. L. Glashow, “Flavor Goniometry By Proton Decay,” Phys. Rev. Lett. 45 (1980) 413.

[34] S. M. Barr, "A New Symmetry Breaking Pattern For SO(10) And Proton Decay,” Phys. Lett. B 112 (1982) 219.

[35] J. P. Derendinger, J. E. Kim and D. V. Nanopoulos, “Anti - SU(5),” Phys. Lett. B 139 (1984) 170.

[36] I. Antoniadis, J. R. Ellis, J. S. Hagelin and D. V. Nanopoulos, "Supersymmetric flipped SU(5) revitalized,” Phys. Lett. B 194 (1987) 231.

[37] J. R. Ellis, D. V. Nanopoulos and J. Walker, "Flipping SU(5) out of trouble," Phys. Lett. B 550 (2002) 99 [arXiv:hep-ph/0205336].

[38] A. Arvanitaki, C. Davis, P. W. Graham, A. Pierce and J. G. Wacker, "Limits on split supersymmetry from gluino cosmology," Phys. Rev. D 72 (2005) 075011 [arXiv:hep-ph/0504210].

[39] I. Dorsner and P. Fileviez Pérez, "Distinguishing between SU(5) and flipped SU(5)," Phys. Lett. B 605 (2005) 391 arXiv:hep-ph/0409095. 